\title{
Characterising electron butterfly pitch angle distributions in the magnetosphere through observations and simulations
}

\author{
M. M. Klida and T. A. Fritz \\ Center for Space Physics, Boston University, 725 Commonwealth Avenue, Boston, MA, USA \\ Correspondence to: M. M. Klida (mklida@bu.edu) \\ Received: 25 May 2012 - Revised: 11 January 2013 - Accepted: 30 January 2013 - Published: 22 February 2013
}

\begin{abstract}
The Imaging Electron Spectrometer (IES) on the Polar satellite has measured the average characteristics of the equatorial electron pitch angle distributions (PADs) in the midnight sector as a function of radial distance out to the $9 R_{\mathrm{E}}$ apogee of the Polar satellite. Depressions in the observed fluxes of electrons occur with pitch angles around $90^{\circ}$ in the equatorial zone, while the more field-aligned electrons remain largely unchanged. The orbital precessions of the satellite have allowed much of the inner equatorial magnetosphere to be observed. Statistically, butterfly PADs with different shapes are observed selectively in different regions, which can provide insight to their source and possible history. Electron paths of varied pitch angles were modelled using Runge-Kutta approximations of the Lorentz force in a Tsyganenko (T96) simulated magnetosphere. The resulting drift paths suggest that the process of magnetopause shadowing plays a significant role in the loss of these electrons. Case studies of the drifting patterns of electrons with varied pitch angles were simulated from Polar's orbit when a butterfly PAD was observed on 3 October 2002 at an altitude near $9 R_{\mathrm{E}}$ and on 12 September 2000 at an altitude near $6 R_{\mathrm{E}}$. These two locations represent regions on each side of the boundary of stable trapping. The modelling effort strongly suggests that magnetopause shadowing does play a significant role in the loss of equatorially drifting electrons from the outer regions of the inner magnetosphere.
\end{abstract}

Keywords. Magnetospheric physics (Energetic particles, trapped; Magnetospheric configuration and dynamics)

\section{Introduction}

A butterfly pitch angle distribution (PAD) exists if a population of particles that have localised minima in the flux of particles that are field-aligned (pitch angles are near $0^{\circ}$ or $180^{\circ}$ ) and equatorially mirroring (pitch angles are near $90^{\circ}$ ). The occurrence of this PAD in the magnetosphere was first described by West (1966), which was a result of upper atmospheric nuclear experimentation in 1962. Since then, the butterfly PAD has been found in naturally occurring populations in both electrons and ions and were reported by Serlemitsos (1966), Pfitzer et al. (1969), Bogott and Mozer (1971), West et al. (1973), West and Buck (1974), Kaye et al. (1978) and Fritz et al. (2003) among others.

The dayside compression of the magnetosphere introduces day/night asymmetries of the magnetic field. This deviation from a dipole results in particles with non-spherical drift shells. As particles that are located in the nightside region of the magnetosphere drift around to the dayside, the resulting drift paths can be roughly characterised by their equatorial pitch angle. By approximately conserving adiabaticity, certain regions of the equatorial nightside will lose particles with pitch angles near $90^{\circ}$ due to contact with the dayside magnetopause. The particles with a longer bounce length will drift around to the dayside more closely to the earth and will not be lost to the magnetopause. The regions of the magnetosphere which contain some particles that are stably trapped while others that will be lost can be called pseudo-trapped or quasi-trapped. These trapping regions are discussed in more detail by Roederer $(1967,1970)$. It is within these regions that a butterfly PAD can be created or maintained.

Due to the orbital precession of the Polar satellite over many years, a large portion of the equatorial magnetosphere inside of $9 R_{\mathrm{E}}$ was sampled. By using the satellite's 


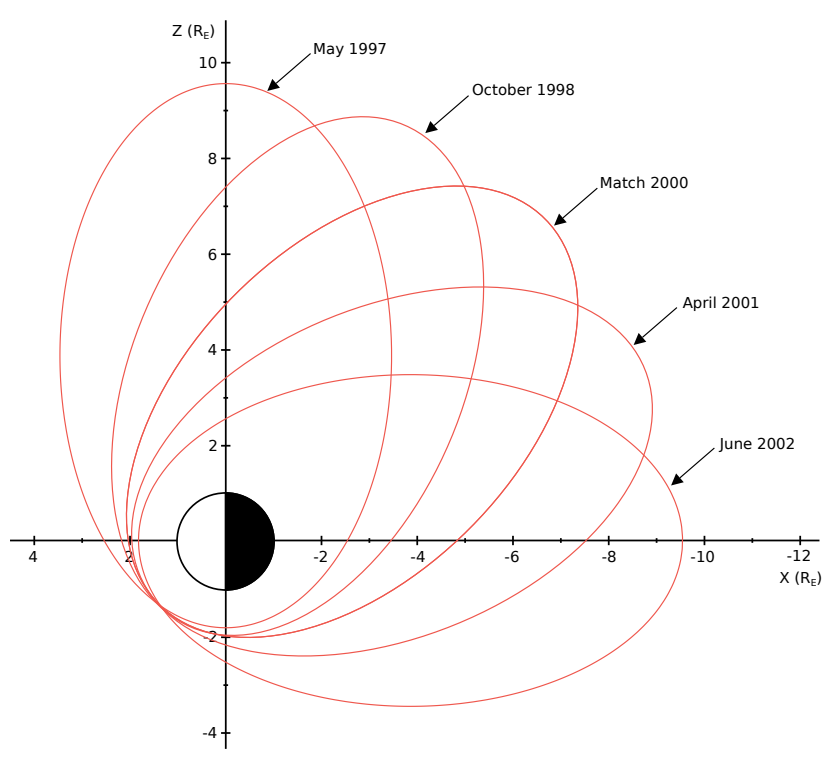

Fig. 1. The precession of the Polar orbit with apogee progressing from the North Pole to the Equator from 1997 to 2002. For purposes of comparison, the plane of the orbits were azimuthally rotated into the $\mathrm{X}-\mathrm{Z}$ plane.

Comprehensive Energetic Particle and Pitch Angle Distribution (CEPPAD) instrument package (Blake et al., 1995) to detect electrons with energies greater than $\sim 30 \mathrm{keV}$, the long-term presence of the butterfly PAD in the nightside equatorial region can be characterised. A particle tracing model can then be used in conjunction with Polar observations to examine the cause and stability of the observed populations.

\section{Polar instrumentation}

Since about 1997, the apogee of the orbit of the Polar satellite has moved from the region above the northern pole down to lower latitudes. This precession of the orbit has consequently caused the magnetic equatorial crossings of the satellite to progress outward from the earth. In addition to this effect, the variation in the local time of the orbit throughout a yearly orbit has allowed for detailed observations across the equatorial plane from less than $3 R_{\mathrm{E}}$ out to almost $10 R_{\mathrm{E}}$. The apogee of the orbit approached the equatorial region in the middle of 2002, signifying the outermost region of the equatorial plane that Polar can measure in situ. Since that time, the magnetic equatorial crossings have travelled inward, as the line of apsides of Polar moved into the Southern Hemisphere. Figure 1 plots the movement of the orbit in the $\mathrm{X}-\mathrm{Z}$ plane from May 1997 until June 2002. For purposes of comparison, all of the orbits have been azimuthally rotated into the noon-midnight plane. The five displayed orbits estimate the latitude at which Polar reached apogee. These orbital characteristics of this satellite make it a valuable resource for sam-

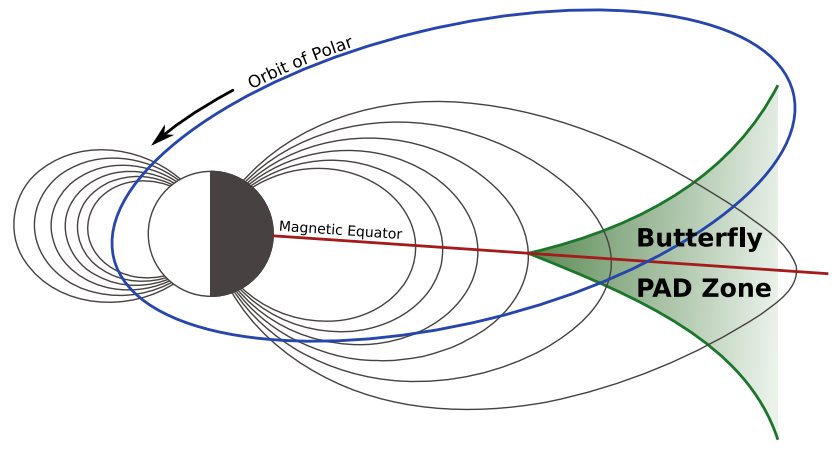

Fig. 2. When Polar passed through the nightside equatorial plane, it was on an outbound trajectory going from the Southern to the Northern Hemisphere into a region where the butterfly PAD is observed.

pling broad regions of the inner magnetosphere including the equatorial plane. A typical Polar orbit through the midnight sector of the equatorial plane is shown in Fig. 2. As Polar crosses the equatorial plane, it is on an outbound trajectory moving from the Southern to the Northern Hemispheres. For many passes through the butterfly PAD region, a wide range of radial distances are sampled in a single orbital pass.

The Imaging Electron Spectrometer (IES) on Polar, a component of CEPPAD, was used to measure high spatial and temporal resolution fluxes of electrons with energies ranging from $30 \mathrm{keV}$ up to $507 \mathrm{keV}$. On the sensor, there were nominally ten energy bins for nine different look direction channels. In total, the instrument could detect electrons with a $180^{\circ}$ polar $\times \sim 20^{\circ}$ azimuthal field of view. The wide polar field-of-view allowed for approximately $4 \pi$ steradian coverage over a complete six second spin period (Blake et al., 1995).

\section{Morphology and classification of the butterfly PAD}

When identifying the presence of butterfly PADs in the Polar observations, the flux is plotted as function of both time and pitch angle. Figures 3 and 4 are a demonstration of observations measured by Polar for 12 September 2000 and 3 October 2002, respectively. Long-term measurements have shown two distinguishable, characteristic shapes to these distributions, a "bowl-shaped" distribution observed in the 12 September example, and a "box-shaped" distribution as shown in the 3 October data. The shape of the butterfly PAD arises from the visual border of depressed flux centred around $90^{\circ}$ pitch angle when plotted in the previously mentioned form. A time evolution in the observations does introduce both a spatial and temporal dependence. However, these dependencies may be characterised with the broad survey of PADs in the nightside equatorial region as discussed in Sect. 4. In addition to these two shapes, there is an additional occurrence of a butterfly PAD that shows hybrid 
Polar IES pitch angle plots (12 September 2000)
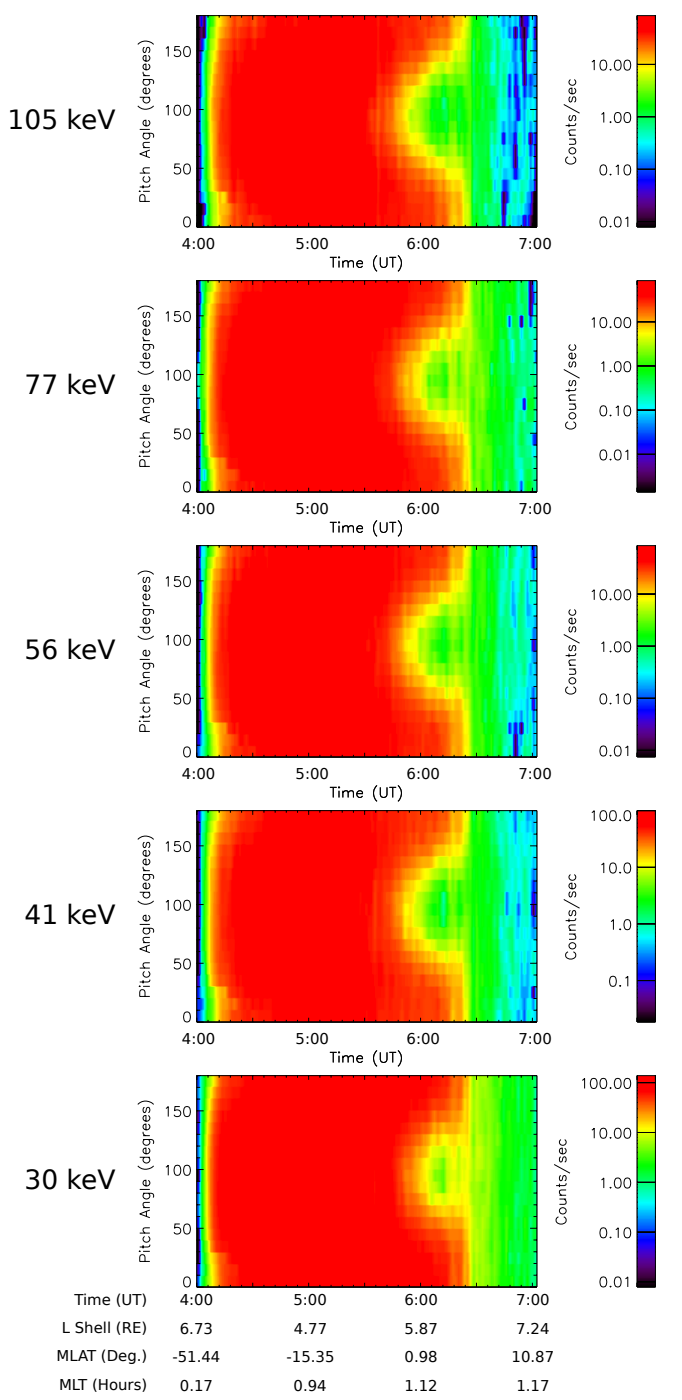
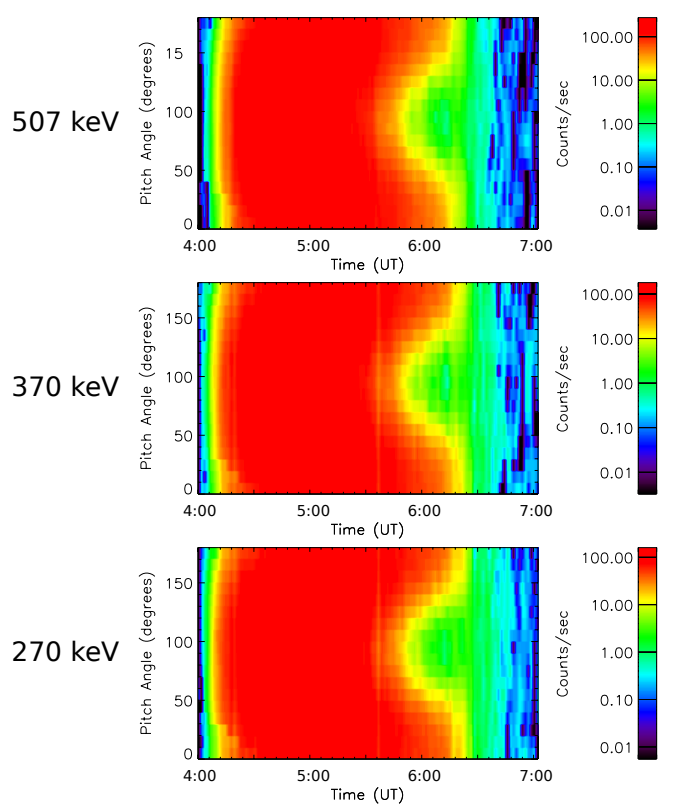

$197 \mathrm{keV}$
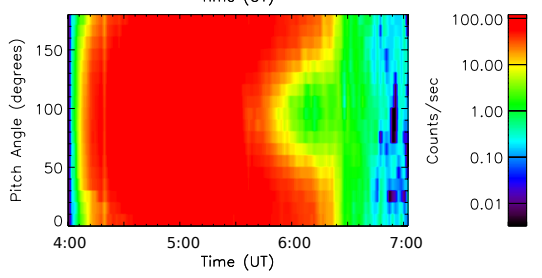

Fig. 3. Electron flux plots from the IES sensor for ten energy channels (indicated by the nominal energy threshold to the left) on Polar for 12 September 2000, with electron flux plotted as functions of pitch angle and time. Butterfly PADs occur between 05:30 UT and 06:30 UT with a bowl-like shape.

characteristics of both the bowl and box-shaped. Further details of these shapes are discussed in the following subsections.

The two plots are flux measurements from Polar as functions of both the electron pitch angle and time for the aforementioned days. In the early years, when the equatorial crossings were closer to the earth, butterfly bite-outs near $90^{\circ}$ were not observed. As the crossings moved outward, the butterfly bite-out was observed by Polar. Slow evolutions in the morphology were also noticed. Early bite-outs, such as 12 September 2000, displayed a bowl-like shape. Later biteouts, such 3 October 2002, displayed a box-like shape, with a rapid onset and return to isotropy. Events in between these two examples can display hybrid characteristics.

\subsection{The bowl-shaped PAD}

The bowl-shaped pitch angle distribution is observed primarily in the inner region of the satellite's magnetic equatorial crossing range. This shape of the distribution is characterised by the onset of the bite-out beginning at a $90^{\circ}$ pitch angle and as Polar passes through the radiation belt region, the flux depression progressively spreads outward to more field-aligned pitch angles. This expansion phase continues until the biteout covers the entire pitch angle range or isotropy is restored to the flux of the distribution.

The time progression of the Polar measurement corresponds to a gradual spatial progression of the width of the butterfly PAD. As Polar enters into the equatorial region from 
Polar IES pitch angle plots (3 October 2002)
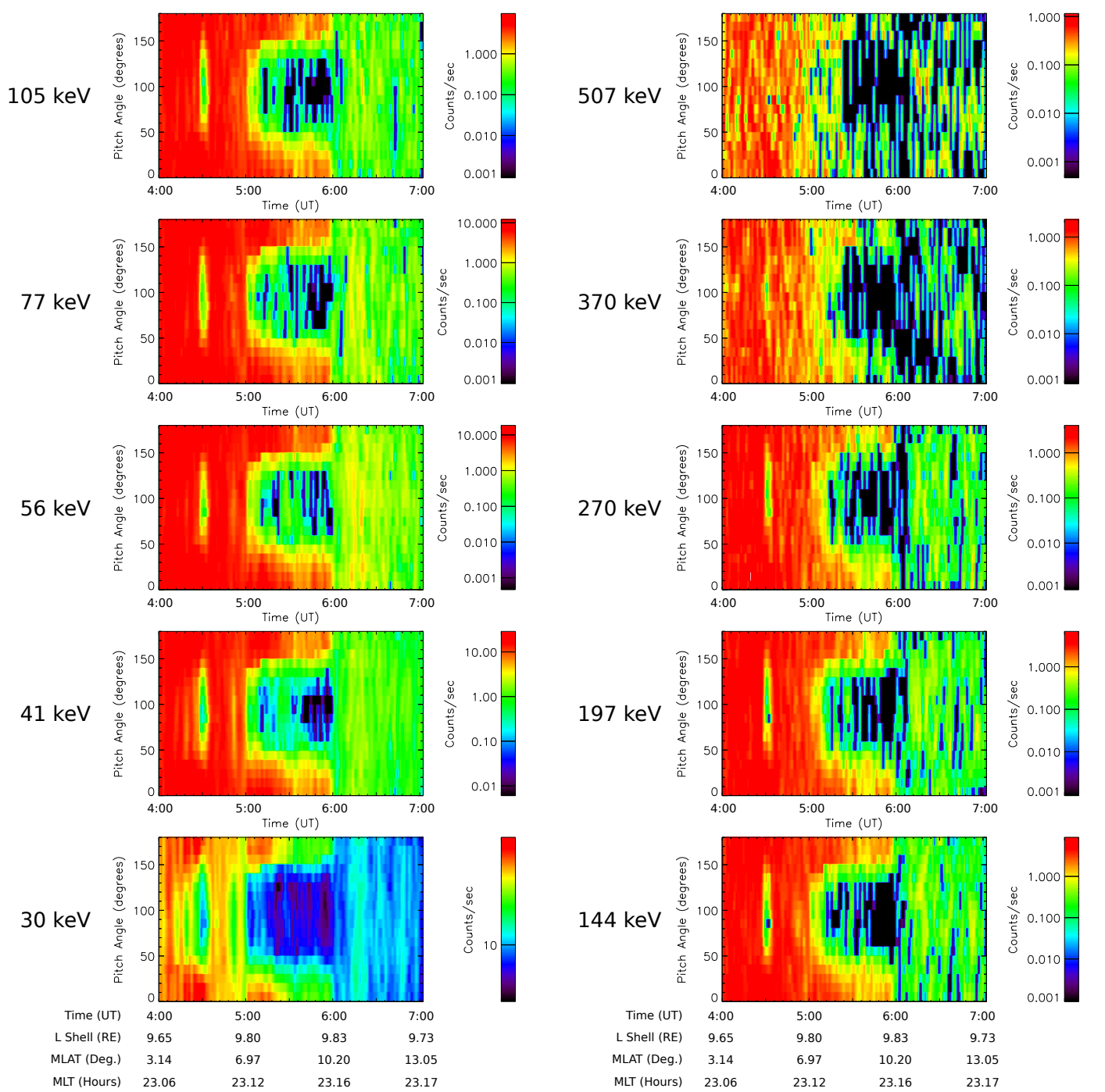

Fig. 4. Electron flux plot from the IES sensor for ten energy channels (indicated by the nominal energy threshold to the left) on Polar for 3 October 2002, with electron flux plotted as functions of pitch angle and time. Butterfly PADs occur between 05:00 UT and 06:00 UT with a more box-like shape.

the Southern Hemisphere, a bowl-shaped butterfly PAD indicates an absence of only locally mirroring $90^{\circ}$ pitch angle particles. Then as Polar continues toward the magnetic equatorial plane, a wider range of pitch angles are lost to the butterfly PAD.

\subsection{The box-shaped PAD}

The box-shaped pitch angle distribution is observed exclusively toward the outer regions of Polar's range of magnetic equatorial crossings. This shape of the distribution is represented by a bite-out onset that initially covers a range of pitch angles centred at $90^{\circ}$. This is a fundamental difference from the "bowl-shaped" distributions as they always begin by the instrument observing a flux decrease only at $90^{\circ}$. The end of the "box-shaped" distribution is a bite-out again with a range of pitch angles centred at $90^{\circ}$, usually followed by an isotropic distribution. In the observations, the width of the bite-out may or may not change as the satellite passes through the equatorial region, but the shape is characterised by the distinct onset and turn-off ranges.

A sharp appearance of a butterfly PAD in the observed Polar pitch angle measurements corresponds to a sharp spatial border between a region in which there is almost a isotropic PAD without any deviations at $90^{\circ}$ pitch angle and a region with a butterfly PAD that shows a depression over a wide range of pitch angles. As Polar passes through the nightside 
equatorial region, the range of pitch angles included in the butterfly PAD may change, but there always exists a significant range of off $90^{\circ}$ particles within the butterfly PAD depression.

\subsection{The hybrid PAD}

Finally, Polar observed butterfly PADs with a hybrid shape. In other words, the beginning and end of the butterfly is able to show different shapes. When apogee is in the Northern Hemisphere, the onset displays bowl-shaped characteristics and the turn-off has box-shaped characteristics. This phenomenon is due to the oblique orbit of the satellite itself. The butterfly shape is observed for an extended period of time, from tens of minutes, up to a few hours. During this prolonged observation, the altitude of Polar can increase significantly. When this occurs, the satellite will move from the bowl-shaped domain out into the box-shaped domain in a single observed butterfly PAD.

After 2002, the apogee of Polar progressed into the Southern Hemisphere, and the hybrids were still observed with regularity. During the period of 2002 until 2003, the orbit still allowed for the satellite to cross the equatorial region as before, except now it is decreasing in altitude. This path transported Polar from the box-shaped region into the bowl-shaped region. Indeed, the hybrid butterfly PADs appear backwards in comparison to the earlier versions, due to the reversal of the temporal sequence for the satellite to encounter these regions.

\section{The statistics of PAD shape on the nightside}

With the observations of the three types of butterfly PAD shape, it is important to determine any patterns that may arise. A survey of Polar's equatorial passes was conducted for August, September and October for the years from 1999 through 2002. These were the months that the orbital apogee was near a local time of midnight. Figure 5 locates the nightside magnetic equatorial crossings made by Polar in these four years. Each point indicates the X and Y GSM coordinates of the crossings. The equatorial crossings of the orbits began lower in altitude and moved outward over time. The number of crossings for each PAD shape is summarised in Table 1 for different ranges of radial distances from the earth.

The presence of the butterfly PAD increased in frequency outside of $5 R_{\mathrm{E}}$. For example, in between $5.5 R_{\mathrm{E}}$ and $9.5 R_{\mathrm{E}}$, a butterfly PAD was observed in approximately $90 \%$ of the equatorial crossings and was observed in at least $80 \%$ of the crossings in each of the $0.5 R_{\mathrm{E}}$ bins. This PAD is a wellestablished feature of the inner magnetosphere. The discrete differences in the butterfly PAD shape allow for a distribution that can characterised as function of radial distance from the earth.

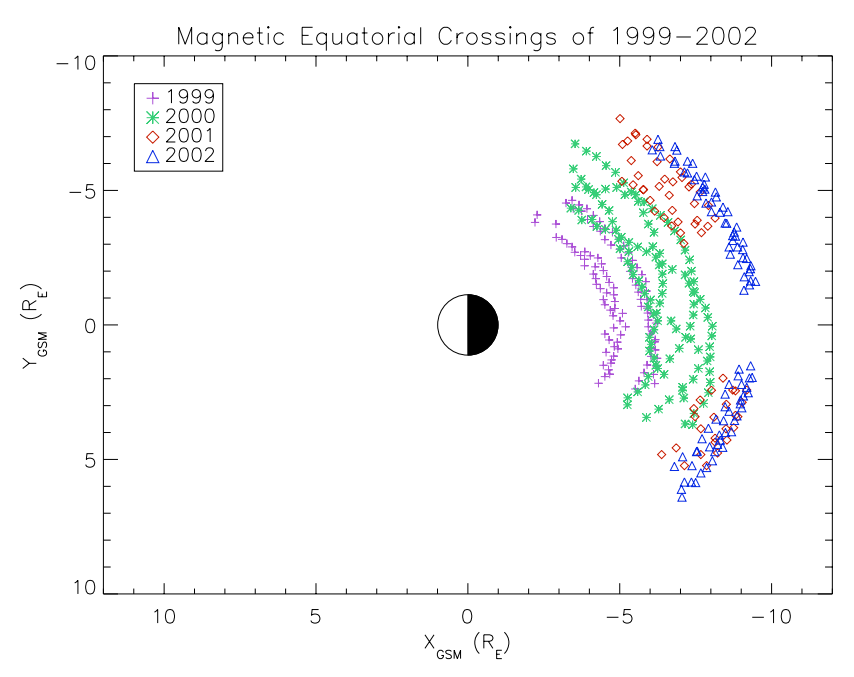

Fig. 5. The locations of the 384 magnetic equatorial crossings that were used for the statistical survey. They are from the months of August, September and October 1999 through 2002.

The resulting bar chart in Fig. 6 indicates the frequency of occurrence of each shape of butterfly PAD. The crossings were grouped into $0.5 R_{\mathrm{E}}$ sections, and the number of occurrences of each shape were compared to the total number of satellite crossings in each section. The bowl-shaped distributions are the dominant type observed toward the inner range of Polar's orbit, and the box-shaped distributions toward the outer range, with a strong population of hybrid distributions in between. With such a strong correlation to the distribution of the shape with respect to the radial distance from the earth, the PAD shapes observed in the Polar pitch angle plots can be attributed primarily to spatial characteristics within the magnetosphere.

\section{Modelling dependencies of electron pitch angle}

Using the Tsyganenko simulated magnetosphere (Tsyganenko, 1995; Tsyganenko and Sitnov, 2005), the differential equation of motion of a particle with mass $m$, and charge $q$ is the Lorentz force,

$m \frac{d \boldsymbol{v}}{d t}=q(\boldsymbol{E}+\boldsymbol{v} \times \boldsymbol{B})$.

The position of the particle has a solution as a function of time and the initial position when given the velocity. These solutions can be numerically estimated to yield a path of motion for electrons with a given initial pitch angle and energy.

Once the magnetic field topology is estimated using the T96 model and the electric field is estimated using the Volland-Stern model (Stern, 1975; Volland, 1978), a RungeKutta type method is used to estimate the Lorentz force ordinary differential equation that governs the motion of the electrons to yield the next point in space in the time sequence. These increments can then be compiled over a time 


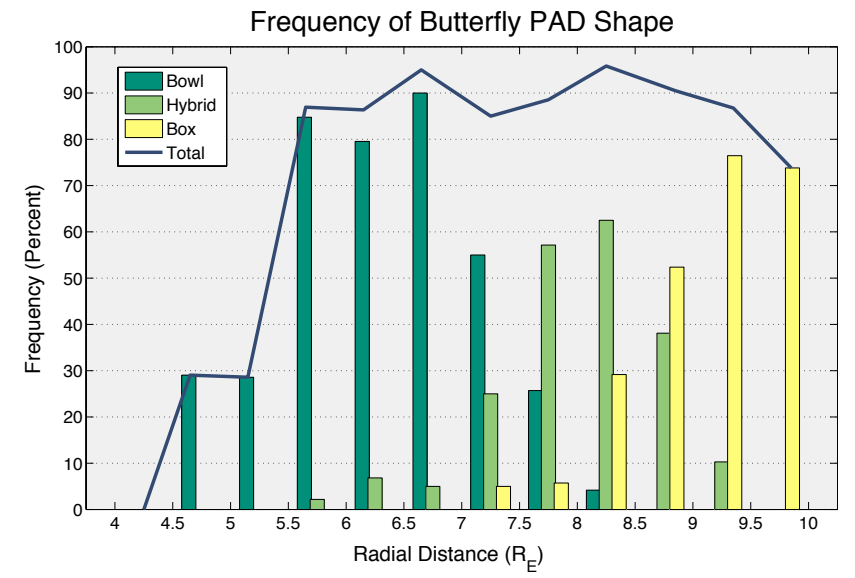

Fig. 6. The distribution of the occurrences of the different shapes of butterfly PAD, according to the radial distance of the magnetic equatorial crossing observed for each event. The distances are separated into $0.5 R_{\mathrm{E}}$ bins and counting statistics are taken within.

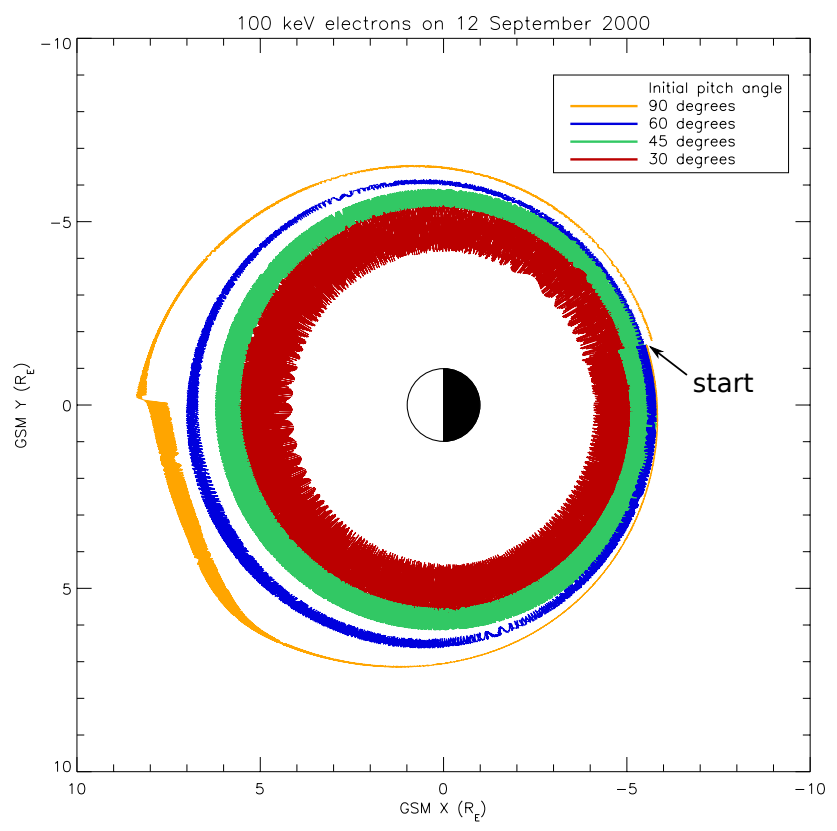

Fig. 7. The differential drift paths of electrons with four different pitch angles are shown. All simulations begin at the location of Polar at 06:00 UT and trace backwards in time until the particle is lost or completes a drift orbit. All four particles drifted around from local noon indicating stably trapped drift orbits.

progression to show the suggested path a particle may take. If modelled backward in time, the model can suggest possible origins.

\subsection{September 2000}

In September 2000, Polar was crossing the magnetic equatorial plane near $6 R_{\mathrm{E}}$. Observations made during this period

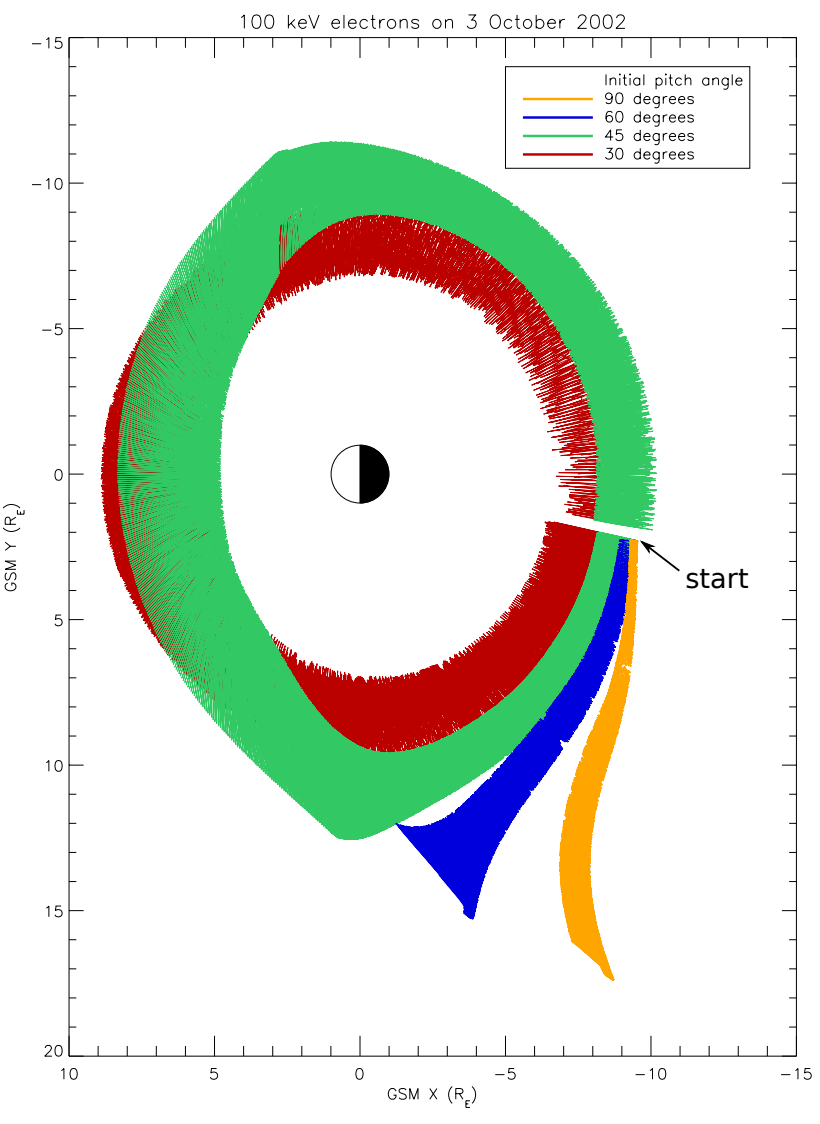

Fig. 8. The differential drift paths of electrons with four different pitch angles are shown. All simulations begin at the location of Polar at 05:00 UT and trace backwards in time until the particle is lost or completes a drift orbit. The paths of the $60^{\circ}$ and $90^{\circ}$ pitch angle electrons are shortened due to contact with the model's magnetopause.

showed either isotropic or butterfly shaped distributions as the satellite crossed the equatorial plane on the nightside.

The data recorded on 12 September 2000 demonstrate a butterfly bite-out with a well defined bowl-like shape, as seen in Fig. 3. The onset of the bite-out begins discretely at $90^{\circ}$ and progressively extends its range until isotropy is restored. The observed bite-out occurred from approximately 05:50 UT until 06:25 UT.

Figure 7 demonstrates the differential drift paths for electrons with different pitch angles. All simulations begin at the location of Polar at 06:00 UT $\left(X=-5.55 R_{\mathrm{E}}, Y=\right.$ $-1.63 R_{\mathrm{E}}$, and $\left.Z=-0.45 R_{\mathrm{E}}[\mathrm{GSM}]\right)$ and trace backwards in time for electrons with four different pitch angles. The one hour average solar wind conditions (from 05:00 to 06:00 UT) used for the model had a solar wind velocity of $361 \mathrm{~km} \mathrm{~s}^{-1}$ and a density of $10.8 \mathrm{~cm}^{-3}$ with an interplanetary magnetic field (IMF) of $B_{\mathrm{y}}=6.4 \mathrm{nT}$ and $B_{\mathrm{z}}=-1.4 \mathrm{nT}$. Also, the Kp index was $3+$ and Dst $=-7$. When Polar observes bite-outs close to the earth (under $6 R_{\mathrm{E}}$ for this example), the differential drift shells do not separate significantly. This allows for 
Table 1. The frequency of Butterfly PAD Shape is characterised by separating all of the crossing statistics into $0.5 R_{\mathrm{E}}$ bins and calculating the occurrence statistics as a function of radial distance. Along with Fig. 6, it can be seen that the bowl and box-shaped butterfly PADs occupy the inner and outer regions, respectively, of the sampled region.

\begin{tabular}{|c|c|c|c|c|c|c|c|c|c|c|c|c|}
\hline \multirow[b]{2}{*}{ Radial distance $\left(R_{\mathrm{E}}\right)$} & \multicolumn{12}{|c|}{ Number of occurrences within each $0.5 R_{\mathrm{E}}$ Bin with centre at radial distance $\left(R_{\mathrm{E}}\right)$} \\
\hline & 4.25 & 4.75 & 5.25 & 5.75 & 6.25 & 6.75 & 7.25 & 7.75 & 8.25 & 8.75 & 9.25 & 9.75 \\
\hline Bowl & 0 & 9 & 4 & 39 & 35 & 18 & 11 & 9 & 1 & 0 & 0 & 0 \\
\hline Hybrid & 0 & 0 & 0 & 1 & 3 & 1 & 5 & 20 & 15 & 8 & 7 & 0 \\
\hline Box & 0 & 0 & 0 & 0 & 0 & 0 & 1 & 2 & 7 & 11 & 52 & 31 \\
\hline No Butterfly & 7 & 22 & 10 & 6 & 6 & 1 & 3 & 4 & 1 & 2 & 9 & 11 \\
\hline Total crossings & 7 & 31 & 14 & 46 & 44 & 20 & 20 & 35 & 24 & 21 & 68 & 42 \\
\hline Butterfly occurrence (\%) & 0.0 & 29.0 & 28.6 & 87.0 & 86.4 & 95.0 & 85.0 & 88.6 & 95.8 & 90.5 & 86.8 & 73.8 \\
\hline
\end{tabular}

the particle tracer to allow all four populations to completely drift around to the dayside, preserving populations from loss to the magnetopause. The asymmetric drift shell of the $90^{\circ}$ electron on the dayside is due to the compression of the magnetosphere from the solar wind which causes minima in the strength of the magnetic field lines to occur off of the equator. It may be concluded that during the observed butterfly PAD, the satellite was inside of the limit of stable trapping and, therefore, magnetopause shadowing during the previous drift orbit is not responsible for the bowl-like butterfly PADs.

\subsection{October 2002}

As time progressed, and the apogee of Polar moved to lower latitude, the equatorial crossings correspondingly moved outward as well, which allowed for the sampling of new regions of the magnetosphere. This example day demonstrates a butterfly bite-out with a well-defined box-like shape. The onset of the bite-out begins immediately for a range of pitch angles and remains constant until isotropy is restored with a sudden drop-off. The observed bite-out occurred from approximately 05:00 UT until 06:00 UT.

Figure 8 demonstrates the differential drift paths for electrons with different pitch angles. All simulations begin at the location of Polar at 05:00 UT ( $X=-9.26 R_{\mathrm{E}}, Y=2.24 R_{\mathrm{E}}$, and $Z=-1.13 R_{\mathrm{E}}$ [GSM]) and trace backwards in time for electrons with four different pitch angles. The one hour average solar wind conditions (from 04:00 to 05:00 UT) used for the model had a solar wind velocity of $481 \mathrm{~km} \mathrm{~s}^{-1}$ and a density of $7.9 \mathrm{~cm}^{-3}$ with an IMF of $B_{\mathrm{y}}=0.8 \mathrm{nT}$ and $B_{\mathrm{z}}=$ $10.5 \mathrm{nT}$. Also, the Kp index was $3-$ and Dst $=-62$. When Polar observes bite-outs farther away from the earth (almost $10 R_{\mathrm{E}}$ for this example), the differential drift shells separate significantly more than the previous, lower altitude example. Electrons with pitch angles near $90^{\circ}$ exhibit sizable lateral drift, which allows for contact with the magnetopause. These drift characteristics allow for the loss of electrons with pitch angles near $90^{\circ}$, creating the well-defined butterfly effect. This means that magnetopause shadowing within the previ- ous drift orbit is most likely responsible for producing the box-like butterfly PADs.

\section{Diffusive effects on butterfly PADs}

The effects of the highly dynamic solar wind on the global structure of the magnetosphere must be considered when analysing the temporal structure of the PADs. When nonadiabatic variations in the magnetosphere occur over short enough time scales, some of the adiabatic invariants may be violated, allowing diffusion mechanisms to occur. Pitch angle and radial diffusion arising from the respective violations in the second and third adiabatic invariants will be examined in the following sections. When this occurs, the population of electrons with a butterfly PAD will encounter pitch angle and radial diffusion.

Although there is a generalised decrease in particle fluxes as the radial distance increases, this is not true for all populations. The scope of work here is electrons with energies in the tens to hundreds of $\mathrm{keV}$. The radial profile of these specific electrons can often be somewhat flat or have a peak near geostationary orbit (Coroniti and Thorne, 1973; Fritz et al., 2003). The shape of the profiles are highly-dependent on the species and energy. Figure 1 from Fritz and Chen (1999) is an exemplification of such a profile measured by the ISEE-1 satellite during a radial pass very close to the magnetic equatorial plane $\left(B / B_{0} \sim 1\right)$ demonstrating these features.

While it is true that the drift shell splitting process would separate co-located dayside particles of different pitch angles, this alone would not produce a butterfly pitch angle distribution. If one were to track a dayside isotropic distribution as it drifts to the nightside, the particles with $90^{\circ}$ equatorial pitch angles would be located at a lesser radial distance than the more field-aligned particles. This would create areas of the equatorial nightside where $90^{\circ}$ particles would be void. This example only includes a dayside population at a single point. Particles with $90^{\circ}$ equatorial pitch angles at radial distances outward of this initial group will fill in the $90^{\circ}$ voids on the nightside that were created by the drift shell splitting of the original population. This effect would 
continue outward until the dayside populations are near the magnetopause. Outside this boundary of stable trapping, the butterfly is caused by magnetopause shadowing via drift shell splitting.

\subsection{Radial diffusion}

The modelling demonstrated a viable loss mechanism to produce box-like butterfly PADs in the outer region of the radiation belts (outside of about $6 R_{\mathrm{E}}$ ), but there was no such mechanism inward of that region. The simulations would suggest that populations of electrons with any pitch angle will drift around the earth in complete and stable orbits. On the other hand, the observed data from Polar indicates that the $90^{\circ}$ pitch angle electrons are also depleted in this region.

This contradiction leaves two possible resolutions to explain butterfly PADs inside of $6 R_{\mathrm{E}}$ : there is either another unknown loss mechanism that is still effective at these lower latitudes, or the distribution are remnants of losses that occurred further outward and have since diffused radially inward. That is, the equatorially mirroring electrons were stripped from the outer populations and then over time, the remaining electrons diffused inward and somewhat preserved their pitch angle distribution.

To test the validity of the second possibility, the rate at which the electrons typically diffuse inward must be examined more rigorously. When the third adiabatic invariant given as,

$\Phi=\oint A_{0} d x$,

where $A_{0}$ is the area encompassed by the drift shell of the particle, is violated, the gradient in the phase space density gives rise to a diffusive force. If the effects of pitch angle scattering are neglected, the force on the particles can be characterised in the form of the radial-diffusion equation (cf., Schulz and Lanzerotti, 1974, Eq. 3.01),

$$
\frac{\partial \bar{f}}{\partial t}=\frac{\partial}{\partial \Phi}\left[D_{\Phi \Phi} \frac{\partial \bar{f}}{\partial \Phi}\right]=L^{2} \frac{\partial}{\partial L}\left[\frac{1}{L^{2}} D_{L L} \frac{\partial \bar{f}}{\partial L}\right] .
$$

This allows for an estimation of the time required for an energetic electron to move inward via a diffusion coefficient $D_{L L}$. In Eq. (3), $\bar{f}$ is the distribution function with respect to the third adiabatic invariant, $|\Phi| \equiv 2 \pi a^{2} B_{0} / L$. For particles mirroring at the equator, the diffusion coefficient can be estimated (cf., Schulz and Lanzerotti, 1974, Sect. III.2) with magnetic spectral densities in a model magnetic field. Additionally efforts to model radial diffusion (e.g., Selesnick et al., 1997; Brautigam and Albert, 2000; Li et al., 2001) can lead to average estimates of the diffusion coefficient and consequent particle transport times. From modelling diffusion for all of 1998, Barker et al. (2005) characterised the electron lifetime as a function of L-shell as $\tau=3(6 / L)^{5.3}$ days. Because of the long timescales over which this expression was determined, it is reasonable to extrapolate this relation to an average expression for all times. This would indicate that on average, electrons in the outer regions of the coverage of Polar (i.e., outside the boundary of stable trapping), would require on the order of a few days to migrate inward to a region where electrons of all pitch angles are stably trapped.

\subsection{Pitch angle diffusion}

A pitch angle distribution on the nightside comprises a population of particles that are not co-located at any other local times, as particles with different pitch angles drift on different shells. It is not appropriate to think of a population of particles drifting around and changing its pitch angle distribution. In a region of stable trapping, a nightside particle will drift around to the dayside and return to the nightside at a roughly similar location and bounce length. If any scattering occurs along the drift orbit, then the bounce length may change and the particle is either lost or it returns to the nightside at a different radial distance. The net result of the pitch angle scattering will be a net loss of electrons and a flattening of the PAD over time at a particular local time.

As the conservation of the second adiabatic invariant, $J$, breaks down, particle mirror points are shifted along field lines. This violation of the second adiabatic invariant,

$$
J=m \oint v_{\|} d s,
$$

where $v_{\|}$is the component of the velocity parallel to the magnetic field line and $d s$ is the particle bounce path, is known as pitch angle diffusion. In other words, $\dot{J} \sim 0$ when $T_{\mathrm{b}} \dot{B} \ll B$, where $T_{\mathrm{b}}$ is the bounce period of the particle.

If the inner butterfly PADs are indeed remnants of those produced at higher altitudes, then the distributions would undergo some degree of pitch angle diffusion while radially diffusing inward over the time-scales described in the previous section. This combination of diffusion processes would then alter the shape of the butterfly PADs. While the distributions are observed in the region of magnetopause shadowing, the borders of the bite-out are constant throughout the region, indicated by the box-shaped distributions that were observed on Polar. However, the older, inner distributions have undergone significant pitch angle scattering since they left the region of magnetopause shadowing. The consequence of this interaction is that the width in pitch angle of the flux depressions centred at $90^{\circ}$ is no longer constant across the butterfly PAD. This effect would lead to a more rounded distribution measured by the Polar satellite, indicated by a bowl-shaped PAD distribution.

Outside the boundary of stable trapping, the butterfly PADs remain uniform throughout the depression, indicated by the box-like shape. The electrons will radially diffuse inward until all particles at certain L-shell can stably drift, regardless of pitch angle. The diffusive effects on the pitch angles of the electrons will then alter the PAD to give a 
bowl-like shape. Because of the orbital geometry of Polar during the studied times, the satellite entered the nightside equatorial zone from the south. As it extended through the equatorial zone where the butterfly PADs occur, it also extended further away from the earth, and the initial narrow onset occurs when Polar was at lower altitude. The narrow pitch angle range of the butterfly PAD is a consequence of longer exposure to pitch angle diffusion. As the butterfly PAD widens out, the depression occurs over a wider range of pitch angles with centre at $90^{\circ}$. This is because these portions of the population have radially diffused inward from the boundary of stable trapping more recently. As a consequence, pitch angle diffusion has had less of an effect than on the more inward electrons. Additionally, when only a portion of the observed butterfly PAD volume is in the stable trapping region, a hybrid shaped distribution can then be measured by Polar.

Pitch angle diffusion will tend to smooth out any features in the PAD, which over time will decrease the severity and spatial extent of the butterfly PAD. The rate of pitch angle diffusion is not constant for all particles, dependent on geomagnetic activity and energy. The pitch angle diffusion constants have a fall off as they approach $90^{\circ}$ pitch angles. While pitch angle diffusion may be quick for long bounce path electrons, equatorially mirroring electrons will not be affected as quickly.

\section{Conclusions}

Across the range of radial distances that Polar crossed the magnetic equatorial plane, the box-like butterfly PADs are predominantly observed farther away from the earth, while the curved bowl-like distributions are almost exclusively observed closer to the earth. The nightside statistics along with the orientation of the hybrid shaped distributions firmly confirm that butterfly PADs are a semi-permanent feature of the magnetosphere. The electrons with pitch angles near $90^{\circ}$ are being lost on a near consistent basis, warranting further examination into their behaviour and any further possible causes of their loss.

The observed shape of the butterfly PAD, whether bowllike, box-like, or a hybrid of the two indicates a fundamental difference in the border between the equatorial butterfly PAD and the higher latitude isotropic PAD. The magnetosphere inside and outside of $8 R_{\mathrm{E}}$ has fundamentally different butterfly PADs.

Particle tracing simulations can confirm the effect of magnetopause shadowing at larger radial distances, causing drifting electrons with pitch angles near $90^{\circ}$ to become lost from their orbits after contacting the magnetopause. However, when simulations are run closer to the earth, the effect diminishes until it is no longer an effective method to cause the butterfly PAD. The simulation shows that drift shell splitting is still occurring, but it was not significant enough to push drifting electrons an adequate distance outward on the dayside to cause any significant losses.

Since butterfly PADs are still observed inward of the stable trapping boundary and away from the effects of magnetopause shadowing, these distributions may likely be the remnants of butterfly PADs that were created on populations of electrons that were originally at greater radial distances and have radially diffused inward. The many drift orbits that the populations would make while diffusing inward would also imply that the electron distributions are only beginning to become isotropic through pitch angle diffusion, indicated by their rounded shape, consequently preserving their distributions longer than previously thought. It may also be possible the magnetopause is just one of multiple loss mechanisms for electrons with pitch angles near $90^{\circ}$, each of which may have a different effectiveness in the various regions of the magnetosphere.

Acknowledgements. The authors wish to thank Brian Walsh for many useful discussions. This research has been supported by NASA grant NNX08AL60G and USAF AFRL contract FA945310-C-0221.

Topical Editor R. Nakamura thanks N. Ganushkina and one anonymous referee for their help in evaluating this paper.

\section{References}

Barker, A. B., Li, X., and Selesnick, R. S.: Modeling the radiation belt electrons with radial diffusion driven by the solar wind, Space Weather, 3, S10003, doi:10.1029/2004SW000118, 2005.

Blake, J. B., Fennell, J. F., Friesen, L. M., Johnson, B. M., Kolasinski, W. A., Mabry, D. J., Osborn, J. V., Penzin, S. H., Schnauss, E. R., Spence, H. E., Baker, D. N., Belian, R., Fritz, T. A., Ford, W., Laubscher, B., Stiglich, R., Baraze, R. A., Hilsenrath, M. F., Imhof, W. L., Kilner, J. R., Mobilia, J., Voss, D. H., Korth, A., Gull, M., Fisher, K., Grande, M., and Hall, D.: Ceppad, Space Sci. Rev., 71, 531-562, 1995.

Bogott, F. H. and Mozer, F. S.: Equatorial electron angular distributions in the loss cone and at large angles, J. Geophys. Res., 76, 6790-6805, doi:10.1029/JA076i028p06790, 1971.

Brautigam, D. H. and Albert, J. M.: Radial diffusion analysis of outer radiation belt electrons during the October 9, 1990, magnetic storm, J. Geophys. Res., 105, 291-310, doi:10.1029/1999JA900344, 2000.

Coroniti, F. V. and Thorne, R. M.: Magnetospheric Electrons, Annu. Rev. Earth Planet. Sci., 1, 107129, doi:10.1146/annurev.ea.01.050173.000543, http: //www.annualreviews.org/doi/abs/10.1146/annurev.ea.01. 050173.000543, 1973.

Fritz, T. A. and Chen, J.: The cusp as a source of magnetospheric particles, Radiation Measurements, 30, 599-608, doi:10.1016/S1350-4487(99)00239-5, http://www.sciencedirect. com/science/article/pii/S1350448799002395, 1999.

Fritz, T. A., Alothman, M., Bhattacharjya, J., Matthews, D. L., and Chen, J.: Butterfly pitch-angle distributions observed by ISEE-1, Planet. Space Sci., 51, 205-219, 2003. 
Kaye, S. M., Lin, C. S., Parks, G. K., and Winckler, J. R.: Adiabatic modulation of equatorial pitch angle anisotropy, J. Geophys. Res., 83, 2675-2682, doi:10.1029/JA083iA06p02675, 1978.

Li, X., Temerin, M., Baker, D. N., Reeves, G. D., and Larson, D.: Quantitative prediction of radiation belt electrons at geostationary orbit based on solar wind measurements, Geophys. Res. Lett., 28, 1887-1890, doi:10.1029/2000GL012681, 2001.

Pfitzer, K. A., Lezniak, T. W., and Winckler, J. R.: Experimental verification of drift shell splitting in the distorted magnetosphere, J. Geophys. Res., 74, 4687-4693, doi:10.1029/JA074i019p04687, 1969.

Roederer, J. G.: On the adiabatic motion of energetic particles in a model magnetosphere, J. Geophys. Res., 72, 981-992, doi:10.1029/JZ072i003p00981, 1967.

Roederer, J. G.: Dynamics of Geomagnetically Trapped Radiation, no. 2 in Physics and Chemistry in Space, Springer-Verlag, Berlin, 1970.

Schulz, M. and Lanzerotti, L. J.: Particle Diffusion in the Radiation Belts, no. 7 in Physics and Chemistry in Space, Springer-Verlag, Berlin, 1974.

Selesnick, R. S., Blake, J. B., Kolasinski, W. A., and Fritz, T. A.: A quiescent state of 3 to $8 \mathrm{MeV}$ radiation belt electrons, Geophys. Res. Lett., 24, 1343-1346, doi:10.1029/97GL51407, 1997.

Serlemitsos, P.: Low-energy electrons in the dark magnetosphere, J. Geophys. Res., 71, 61-77, doi:10.1029/JZ071i001p00061, 1966.

Stern, D. P.: Charged particle motions in a magnetic field that reduce to motions in a potential, Am. J. Phys., 43, 689-694, doi:10.1119/1.9714, 1975 .
Tsyganenko, N. A.: Modeling the Earth's magnetospheric magnetic field confined within a realistic magnetopause, J. Geophys. Res., 100, 5599-5612, doi:10.1029/94JA03193, 1995.

Tsyganenko, N. A. and Sitnov, M. I.: Modeling the dynamics of the inner magnetosphere during strong geomagnetic storms, J. Geophys. Res., 110, A03208, doi:10.1029/2004JA010798, 2005.

Volland, H.: A model of the magnetospheric electric convection field, J. Geophys. Res., 83, 2695-2699, doi:10.1029/JA083iA06p02695, 1978.

West, Jr., H. I.: Some observations of the trapped electrons produced by the Russian high-altitude nuclear detonation of October 28, 1962, in: ASSL Vol. 5: Radiation Trapped in the Earth's Magnetic Field, edited by: McCormac, B. M., pp. 634-662, 1966.

West, Jr., H. I. and Buck, R. M.: Pitch angle distributions of energetic electrons in the equatorial regions of the outer magnetosphere - OGO-5 observations, in: Magnetospheric physics: proceedings of the Advanced Summer Institute held at Sheffield, UK, August 1973, edited by: McCormac, B. M., pp. 93-104, D. Reidel Publishing Co., Dordrecht, 1974.

West, Jr., H. I., Buck, R. M., and Walton, J. R.: Electron Pitch Angle Distributions throughout the Magnetosphere as Observed on Ogo 5, J. Geophys. Res., 78, 1064-1081, doi:10.1029/JA078i007p01064, 1973. 